\title{
Native-valve endocarditis due to Candida pulcherrima
}

\section{Antonella D'Andria}

Servizio di M edicina di Laboratorio

A.O. "S. Giovanni di Dio e Ruggi d'Aragona" - Salerno

Key Words Endocarditis, C. pulcherrima, blood culture

\section{Endocardite da C. pulcherrima su valvola aortica nativa in soggetto tossicodipendente}

\section{SUMMARY}

Fungal endocarditis (FE) is an uncommon disease, an while accounting for only 1.3-6\% of all cases of infectious endocarditis, it carries a high mortality risk. U sually occurring in intravenous-drug abusers and prosthetic valve recipients, native-valve endocarditis is rarely reported.

Fungal endocarditis in commonly complicated by systemic embolization, and the difficulty in isolating the fungi with routine blood cultures complicates the diagnostic process. In these culture-negative cases of endocarditis, etiologic diagnosis is made with histolopathologic examination of the cardiac valve, embolic materials, and systemic ulcers. The fungal endocarditis is considered an absolute indication for valve replacement.Although Candida albicans represents the main etiology of fungal endocarditis, Candida parapsilosis is the most common non-albicans species.

The incidence of endocarditis produced by the so-called "opportunists" is progressively increasing in frequency and gradually transforming the clinical picture habitually associated with this disease.

We describe a first case of Candida pulcherrima endocarditis involving the aortic valves, with the patient not survived.

Received June 29, 2009

Accepted August 22, 2009

\section{INTRODUZIONE}

Le endocarditi da miceti (FE) sono patologie molto rare associate ad elevata mortalità (50\%) (3). I dati più recenti indicano che le FE rappresentano solo il 1.3-6\% dei casi di endocardite infettiva (4). Solitamente la diagnosi di FE viene fatta in soggetti dediti all'abuso di droga per via endovenosa portatori di impianti cardiaci protesici o portatori di cateteri vascolari a permanenza. Molto rara l'incidenza di FE in valvola nativa, tipica di portatori di valvulopatie.

Le FE si manifestano in genere con vegetazioni più estese di quelle che si manifestano nelle endocarditi batteriche, presentano uno spiccato tropismo per le valvole, distruggono i tessuti adiacenti e promuovono la formazione di emboli settici. Più frequentemente le FE coinvolgono le valvole aortica e mitralica.

La diagnosi di FE è frequentemente complicata e tardiva, sia perchè molti dei segni clinici e dei sintomi non sono specifici, sia perchè le routinarie emocolture risultano solitamente negative. Nei casi di endocardite ad emocoltura-negativa, la dia- gnosi etiologica viene frequentemente realizzata mediante esame colturale e istopatologico delle valvole cardiache, degli emboli settici e delle eventuali ulcere sistemiche evidenziate nel paziente.

In caso di FE il trattamento farmacologico con antimicotici può non essere sufficiente, infatti in questi casi trova assoluta indicazione il trattamento chirurgico.

C. albicans è il micete che più frequentemente è risultato responsabile di questa rara patologia, mentre tra le candide non-albicans, $C$. parapsilosis è quella più frequentemente riscontrata. La terapia tradizionale per le endocarditi da candida non-albicans prevede amphotericina $\mathrm{B}$ per via endovenosa, con o senza fluconazolo, la terapia chirurgica di sostituzione valvolare è assolutamente indicata.

Descriviamo il primo caso di endocardite da una candida non-albicans, Candida pulcherrima, riconosciuta patogeno emergente tra i lieviti nel 1995 (5). In questo lavoro riportiamo il caso di un paziente tossicodipendente affetto da endocardite

\section{Corresponding author: D'Andria Antonella}

Servizio di Medicina di Laboratorio A.O. "S. Giovanni di Dio e Ruggi d'Aragona"

Via San Leonardo - 84100, Salerno - Tel.: 089672582

E-mail: antoniad@alice.it 
infettiva complicata su valvola aortica bicuspide e deceduto per arresto cardiocircolatorio dopo intervento di splenectomia. La diagnosi di endocardite da C. pulcherrima è stata fatta post-mortem grazie all'esame colturale eseguito su frammenti provenienti da ascesso splenico.

\section{Descrizione del caso}

Caso clinico: Paziente maschio di 47 anni giunge al pronto soccorso nell'ottobre 2007, per dolore toracico acuto, dispnea marcata e febbre persistente da circa 2 mesi. Il paziente riferisce una storia ventennale di tossicodipendenza, epatopatia cronica da HCV e da circa 8 otto anni è nota una bicuspidia aortica con dilatazione della aorta ascendente.

$\mathrm{Al}$ pronto soccorso viene eseguita una angio TC aorta torace-addome che conferma la dilatazione asimmetrica dell'aorta ascendente, rivela un versamento pleurico bilaterale, un versamento pericardico, epatosplenomegalia con ipodensità del polo inferiore della milza posteriore. Il paziente viene ricoverato presso l'UTIC con la diagnosi di dolore toracico in aneurisma aorta ascendente. $\mathrm{Al}$ ricovero vengono eseguite emocolture seriate (3 set) e indagini di laboratorio che evidenziano una anemia di grado lieve, leucociti e formula nella norma, transaminasi nella norma, un lieve aumento della Troponina I, un aumento degli indici flogistici e una ipergammaglobulinemia, Tabella 1. Viene inoltre evidenziata una onicomicosi al $1^{\circ}$ dito del piede destro su cui purtroppo, non si ritiene di dover procedere a indagini colturali.

Tabella 1. Parametri ematochimici riscontrati nel paziente al ricovero

\begin{tabular}{ll}
\hline Globuli rossi & $3.5 \times 10^{3} / \mathrm{uL}$ \\
\hline Globuli bianchi & $6.8 \times 10^{3} / \mathrm{uL}$ \\
\hline Neutrofili & $5.05 \times 10^{3} / \mathrm{uL}$ \\
\hline Piastrine & $161.0 \times 10^{3} / \mathrm{uL}$ \\
\hline VES & $52 \mathrm{~mm}$ \\
\hline PCR & $2.7 \mathrm{mg} / \mathrm{dL}$ \\
\hline GOT & $50 \mathrm{U} / \mathrm{L}$ \\
\hline GPT & $43 \mathrm{U} / \mathrm{L}$ \\
\hline CPK & $56 \mathrm{U} / \mathrm{L}$ \\
\hline CTNI & $0.60 \mathrm{ng} / \mathrm{ml}$ \\
\hline Mioglobina & $40 \mathrm{ng} / \mathrm{ml}$ \\
\hline Gamma & $39.9 \mathrm{~g} / \mathrm{dL}$ \\
\hline
\end{tabular}

$\mathrm{Al}$ ricovero vengono anche eseguite ecocardiografia transtoracica e transesofagea che confermano la bicuspidia della valvola aortica e rivelano anche che questa è diffusamente e severamente calcifica, senza tuttavia evidenza di masse endocarditiche ma con sospetta perforazione del lembo posteriore. L' ecocardiografia transesofagea rivela che il lembo posteriore della valvola aortica pre- senta una soluzione di continuo in prossimità della giunzione mitro-aortica da verosimile rottura. In corrispondenza della giunzione mitro-aortica è presente un grosso pseudoaneurisma.

Vi è una insufficienza aortica massiva. La valvola mitrale e la tricuspide mostrano una insufficienza di grado moderato.

L' ecografia splenica evidenzia un tessuto disomogeneo con lesione ascessuale nel polo inferiore della milza posteriore.

Dopo consulenza infettivologica viene somministrata terapia antibiotica ad ampio spettro (Ampicillina 2 fl ogni 8 h; Netilmicina 300 gm x24h).

In sintesi il paziente risulta affetto da uno stato settico secondario ad endocardite infettiva su valvola aortica bicuspide complicata da:

- rottura del lembo posteriore della valvola aortica, - pseudoaneurisma della giunzione mitro-aortica, - aneurisma dell'aorta ascendente,

- scompenso acuto,

- probabili emboli splenici,

- epatopatia cronica HCV correlata complicata da vasculopatia agli arti inferiori.

A quindici giorni dal ricovero una nuova ecografia splenica conferma l'ascesso e, in conseguenza di ciò, viene disposto un intervento di splenectomia. Tre piccoli frammenti della milza provenienti dalla lesione ascessuale vengono inviati in laboratorio per la coltura. Sei ore dopo l'intervento e a 18 giorni dal ricovero si ha l'exitus del paziente per arresto cardiaco.

\section{Microbiologia}

Le emocolture (5 set eseguiti nell' arco di 10 giorni: 3 set all'ingresso prima che venisse praticata terapia antibiotica e 2 set dopo 10 giorni quando al paziente veniva già somministrata terapia con antibiotici) e le colture dei tre frammenti di milza provenienti dall'ascesso splenico, sono state processate con sistema Bactec9240 (Becton Dickinson). Per le emocolture viene routinariamente applicato un protocollo di 7 giorni, per le brodo-colture un protocollo di 4 giorni.

Dai brodi risultati positivi vengono allestiti preparati per l'esame batterioscopico a fresco e con colorazione Gram. Le subcolture allestite routinariamente su 5\% Sheep Blood Agar, Mannitol Salt Agar, Mac Conkey Agar e Agar Sabouraud.

I test biochimici eseguiti con sistema VITEK2 card Yeast (ID-YST). La card per l'identificazione dei lieviti ID-YST è indicata per l'identificazione dei lieviti più significativi dal punto di vista clinico. La card ID-YST si basa su metodi biochimici sperimentati e contiene 46 test biochimici. 
I 5 set di emocolture sono risultati tutti negativi. Sono invece risultate tutte positive dopo 24 ore di coltura le tre brodocolture allestite con i frammenti provenienti dall'ascesso splenico. L'esame batterioscopico a fresco ha mostrato lo sviluppo di lieviti e le subcolture realizzate anche su Sabouraud Agar hanno mostrato, dopo 24 ore di coltura, lo sviluppo di colonie alcune delle quali caratterizzate da un peculiare pigmento rossomattone. Le prove biochimiche hanno identificato il lievito come Candida pulcherrima.

Gli esami isto-patologici eseguiti post-mortem hanno confermato la diagnosi di endocardite da miceti.

\section{Commento}

L'incidenza delle endocarditi da miceti, a esito infausto si osserva in percentuali importanti nei soggetti dediti all'uso di stupefacenti (12-4\% e soprattutto a livelli delle sezioni cardiache di destra), nei pazienti con protesi valvolari (fase post operatoria precoce) e nei pazienti con anomalie cardiache congenite (rispettivamente nel 40 e $47 \%$ dei casi di FE). I miceti più comunemente in causa sono la C. albicans, le specie non-albicans, l'Aspergillus fumigatus e la Torulopsis glabrata.

Le FE nei pazienti immunocompromessi, possono frequentemente rappresentare la complicanza di prolungati trattamenti antibiotici in infusione e/o anche di procedure non invasive quali l'alimentazione parenterale. La loro localizzazione è più frequentemente murale, molto più rara su valvole native.

La caratteristica clinica delle FE è la tendenza alla formazione di ampie vegetazioni ad elevato rischio embolico. Tali fenomeni di embolizzazione ne condizionano il decorso clinico.

Il trattamento farmacologico, che prevede la somministrazione di Amphotericina B o Fluconazolo, presenta assai scarse possibilità di eradicare l'infezione. La mortalità a breve termine con il solo trattamento antifungino è elevatissima e superiore all' $80 \%$ dei casi. Per questo motivo il trattamento radicale prevede la resezione chirurgica della massa vegetante associato a prolungata terapia con antimicotici in infusione. La tempestività della diagnosi e della terapia chirurgica in questi pazienti condiziona significativamente i risultati a breve termine e lungo termine.

Nel caso presentato è assai verosimile che il decorso sfavorevole trovi la sua principale causa nella mancata tempestiva diagnosi, ma probabili concause sono da ricercare nelle pessime condizioni cliniche e soprattutto nello stato di immunocompromissione del paziente al ricovero.

Con il presente lavoro si vuole, da un lato, ribadire il concetto oramai noto che nei pazienti tossicodipendenti con quadro clinico ed ecocardiografia suggestiva per endocardite complicata, la negatività delle emocolture deve sempre proporre ipotesi di infezione da miceti; e, dall'altra, si vuole confermare che si sta assistendo ad un aumento della lista dei possibili patogeni di interesse clinico. Sulla scorta di questa esperienza proponiamo un network con laboratori a più elevato contenuto tecnologico: una rete di lavoro che consenta la identificazione e lo studio di patogeni emergenti.

\section{BIBLIOGRAFIA}

1. Diekema DJ, Messer SA, Hollis RJ, et al. An outbreak of Candida parapsilosis prosthetic valve endocarditis. Diagn Microbiol Infect Dis. 1997;29:147-153

2. Lejko-Zupanc T, Kozelj M. A case of recurrent Candida parapsilosis prosthetic valve endocarditis: cure by medical treatment alone. $J$ Infect. 1997;35:81-82

3. Muehrcke DD, Lytle BW, Cosgrove DM 3rd. Surgical and long-term antifungal therapy for fungal prosthetic valve endocarditis. Ann Thorac Surg. 1995;60:538-543

4. Saito Y, Takahashi M, Sato A, et al. Isolated tricuspid valve endocarditis due to Candida parapsilosis associated with long-term central venous catheter implantation. Intern Med. 2001;40:403-404

5. Thakur RK, Skelcy KM, Kahn RN, et al. Successful treatment of Candida prosthetic valve endocarditis with a combination of fluconazole and amphotericin B. Crit Care Med. 1994;22:712-714;

6. Trizna Z, Chen SH, Lockhart S, et al. Candida parapsilosis chrondritis successfully treated with oral fluconazole. Arch Dermatol. 2000;136:804 